\title{
GPs could screen for gum disease in people who fail to visit dentists
}

People who do not see a dentist regularly could be screened for gum disease by their GP instead to ensure they are treated quickly and to prevent problems worsening, suggests a study ${ }^{1}$ published recently in the Journal of Clinical Periodontology, the official publication of the European Federation of Periodontology.

Spanish and US researchers used a screening tool in primary care settings to identify patients who may need to be referred for periodontal examination for the study published on 20 March 2019.

About half of people over 30 years of age have periodontitis, which if untreated, can cause tooth loss and inflammation throughout the body, which has been linked with higher risks of diabetes, heart attack and stroke.

The researchers set out to develop and validate a predictive model for moderate-to-severe periodontitis in the adult USA population, using data from the 2011-2012 National Health and Nutrition Examination Survey (NHANES) cycle.

The model was developed and tested in 3,017 adults over 30 years of age with more than 14 teeth from the NHANES.
These people had received a periodontal examination in addition to data collected on cardio-metabolic risk measures (smoking habit, body mass index [BMI], blood pressure, total cholesterol and glycated haemoglobin).

Around half had moderate-to-severe periodontitis, with $13 \%$ having severe and $37 \%$ having moderate periodontitis.

The study was a collaboration between the University Complutense of Madrid, the Forsyth Institute, and the Harvard School of Dental Medicine.

Based on the model, the lowest relative risk (RR) of moderate-to-severe periodontitis corresponded to a non-Hispanic white female, non-smoker, aged 30-40 years, with normal blood sugar. The highest risk - nearly ten-fold greater - corresponded to a Hispanic male smoker, aged 70-80 years, with high blood glucose.

Dr Eduardo Montero, of the Complutense University of Madrid, and first author of the study, said: 'Periodontitis is one of the most prevalent non-communicable diseases, with direct impacts on oral and general health.

'Many patients are undiagnosed because they don't see a dentist. In our study, more than $40 \%$ of adults had not seen a dentist in the past year. GP appointments are more common in some countries, so we created a screening tool with five pieces of routinely collected information to identify people at risk of periodontitis who should be referred to a dentist for diagnosis and treatment if needed.'

Dr Corneliu Sima, Assistant Professor of Oral Medicine, Infection and Immunity, Harvard School of Dental Medicine, Boston, and director of the study, said: 'There is a need for clinical decision support tools to better integrate oral and medical care worldwide, improve quality of life, and reduce healthcare costs.'

Dr Montero added: 'Screening for periodontitis in primary care could have a big impact on public health. Treatment of periodontitis improves oral health, helps people with diabetes control their blood sugar, and reduces systemic inflammation and other factors indirectly associated with the development of clogged arteries (atherosclerosis).'

\section{References}

1. Montero E, Herrera D, Sanz M, Dhir S, Van Dyke T, Sima C. Development and validation of a predictive model for periodontitis using NHANES 2011-2012 data. J Clin Periodontol 2019; 00: 1-10.

\section{Mouth cancer charity goes from strength to strength}

Mouth cancer charity Let's Talk About Mouth Cancer is on track for another successful year following successful campaigning, fund raising and plans to host a global oral event.

The charity took part in Mouth Cancer Action Week in the city of Dundee in November 2018 to reach out and spread the word to a variety of at-risk communities by teaming up with dental students from the University of Dundee.

The main campaign week 19-23 November 2018 began with two days of action with final year dental students. In the morning sessions, interactive learning tutorials and lectures equipped the students with the knowledge and confidence in mouth cancer prevention, detection, diagnosis and patient empowerment.

The Action Day afternoons were spread out across Dundee and Angus where small teams of students and dental core trainees went out to targeted high risk groups with tailored messages about mouth cancer risk and detection.

The groups reached were ethnic minority women at the Dundee International Women's Centre, care workers at multiple local residential elderly care homes, and young people on the university campus.

These teams used their knowledge to advise the target groups about how to identify common early signs and symptoms of mouth cancer in their own or their care home residents' mouths.

During the awareness week, Dundee Dental Students Society also ran lunchtime stalls on the university campus to reach out to other young people and managed to raise more than $£ 900$ by a combination of raffles, bake sales and a music event.

Let's Talk About Mouth Cancer is celebrating its fifth birthday this year and is now preparing to host the Global Oral Cancer Forum (GOCF) in Edinburgh on 6 and 7 March 2020.

More information on the charity is available at www.ltamc.org. 